\title{
Bose condensation in (random) traps
}

\author{
Th. Jaeck ${ }^{1}$, J.V. Pulé1, V.A. Zagrebnov² \\ ${ }^{1}$ School of Mathematical Sciences, University College Dublin Belfield, Dublin 4, Ireland \\ 2 Université de la Méditerranée (Aix-Marseille II), Centre de Physique Théorique - UMR 6207 CNRS, \\ Luminy - Case 907, 13288 Marseille, Cedex 09, France
}

Received June 15, 2009

We study a non-interacting (perfect) Bose-gas in random external potentials (traps). It is shown that a generalized Bose-Einstein condensation in the random eigenstates manifests if and only if the same occurs in the one-particle kinetic-energy eigenstates, which corresponds to the generalized condensation of the free Bose-gas. Moreover, we prove that the amounts of both condensate densities are equal. This statement is relevant for justification of the Bogoliubov approximation in the theory of disordered boson systems.

Key words: generalized Bose-Einstein condensation, random potentials, integrated density of states, Lifshitz tails

PACS: 05.30.Jp, 03.75.Fi, 67.40.-w

\section{Introduction}

The problem of the occurrence of the Bose-Einstein Condensation (BEC) in random media was considered for the first time in the papers by Kac and Luttinger, see [1,2], and then by Luttinger and Sy [3]. In the latter reference, the authors studied a non-interacting (perfect) one- dimensional system with point impurities distributed according to the Poisson law. The authors conjectured a macroscopic occupation of the random ground state, but this was not rigorously proved until $[5,6]$. Although the free Bose-gas (i. e., the perfect gas without external potential or traps) does not exhibit BEC for dimension less than three, the randomness can enhance BEC even in one dimension, see [4]. This striking phenomenon is a consequence of the exponential decay of the one particle density of states at the bottom of the spectrum, known as Lifshitz tails, or "doublelogarithmic" asymptotics, which is generally believed to be associated with the existence of localized eigenstates [12].

On the other hand, the BEC is usually associated with a macroscopic occupation of the lowest one-particle kinetic-energy eigenstates, which are spatially extended (plane) waves. Therefore, it is not immediately clear whether the phenomenon discovered in random boson gases, i. e. macroscopic occupations of localized one-particle states, has any relation to the standard BEC. This is of particular interest in view of the well-known Bogoliubov approximation [7] and its applications to disordered boson systems, see e. g. [8,9], where the a priori assumption of the momentum-space condensation is essential, and is far from trivial to check.

We prove that for the perfect Bose-gas and for a general class of non-negative random potentials, the condensation in the random localized one-particle states and the generalized van den Berg-Lewis-Pulé condensation of bosons in the lowest one-particle kinetic-energy states occur simultaneously, and, moreover, the densities of the condensate fractions are equal. Our line of reasoning is also applicable to some non-random potentials, for example to the case of a perfect gas in weak (scaled) traps studied in [18].

\section{Model, notations and definitions}

Let $\left\{\Lambda_{l}:=(-l / 2, l / 2)^{d}\right\}_{l \geqslant 1}$ be a sequence of hypercubes of side $l$ in $\mathbb{R}^{d}, d \geqslant 1$, centered at the origin of coordinates with volumes $V_{l}=l^{d}$. We consider a system of identical bosons, of mass $m$, 
contained in $\Lambda_{l}$. For simplicity, we use a system of units such that $\hbar=m=1$. First we define the self-adjoint one-particle kinetic-energy operator of our system by:

$$
h_{l}^{0}:=-\frac{1}{2} \Delta_{\mathrm{D}}
$$

acting in the Hilbert space $\mathscr{H}_{l}:=L^{2}\left(\Lambda_{l}\right)$. The subscript $D$ stands for Dirichlet boundary conditions. We denote by $\left\{\psi_{k}^{l}, \varepsilon_{k}^{l}\right\}_{k \geqslant 1}$ the set of normalized eigenfunctions and eigenvalues corresponding to $h_{l}^{0}$. By convention, we order the eigenvalues (counting the multiplicity) as $\varepsilon_{1}^{l} \leqslant \varepsilon_{2}^{l} \leqslant \varepsilon_{3}^{l} \ldots$.

We define an external random potential $v^{(\cdot)}(\cdot): \Omega \times \mathbb{R}^{d} \rightarrow \mathbb{R}, x \mapsto v^{\omega}(x)$ as a random field on a probability space $(\Omega, \mathcal{F}, \mathbb{P})$, satisfying the following conditions:

(i) $v^{\omega}, \omega \in \Omega$, is non-negative;

(ii) $p:=\mathbb{P}\left\{\omega: v^{\omega}(0)=0\right\}<1$.

As usual, we assume that this field is regular, homogeneous and ergodic. Then the corresponding random Schrödinger operator acting in $\mathscr{H}:=L^{2}\left(\mathbb{R}^{d}\right)$ is a perturbation of the kinetic-energy operator:

$$
h^{\omega}:=-\frac{1}{2} \Delta+v^{\omega}
$$

defined as a sum in the quadratic-forms sense. The restriction to the box $\Lambda_{l}$, is specified by the Dirichlet boundary conditions and for regular potentials one gets the self-adjoint operator:

$$
h_{l}^{\omega}:=\left(-\frac{1}{2} \Delta+v^{\omega}\right)_{D}=h_{l}^{0} \dot{+} v^{\omega},
$$

acting in $\mathscr{H}_{l}$. We denote by $\left\{\phi_{i}^{\omega, l}, E_{i}^{\omega, l}\right\}_{i \geqslant 1}$ the set of normalized eigenfunctions and corresponding eigenvalues of $h_{l}$. Again, we order the eigenvalues (counting the multiplicity) so that $E_{1}^{\omega, l} \leqslant$ $E_{2}^{\omega, l} \leqslant E_{3}^{\omega, l} \ldots$. Note that the non-negativity of the random potential implies that $E_{1}^{\omega, l}>0$. So, for convenience we also assume that in the thermodynamic limit almost surely (a. s.) with respect to the probability $\mathbb{P}$, the lowest edge of this random one-particle spectrum is:

(iii) a. s. $\lim _{l \rightarrow \infty} E_{1}^{\omega, l}=0$.

When no confusion arises, we shall omit the explicit mention of $l$ and $\omega$ dependence. Note that the non-negativity of the potential implies that:

(a) $Q\left(h_{l}^{\omega}\right) \subset Q\left(h_{l}^{0}\right), \quad Q$ being the quadratic form domain,

(b) $\left(\varphi, h_{l}^{\omega} \varphi\right) \geqslant\left(\varphi, h_{l}^{0} \varphi\right), \forall \varphi \in Q\left(h_{l}^{\omega}\right)$.

Now, we turn to the many-body problem. Let $\mathscr{F}_{l}:=\mathscr{F}_{l}\left(\mathscr{H}_{l}\right)$ be the symmetric Fock space constructed over $\mathscr{H}_{l}$. Then $H_{l}:=\mathrm{d} \Gamma\left(h_{l}^{\omega}\right)$ denotes the second quantization of the one-particle Schrödinger operator $h_{l}^{\omega}$ in $\mathscr{F}_{l}$. Note that the operator $H_{l}$ acting in $\mathscr{F}_{l}$ has the form:

$$
H_{l}=\sum_{i \geqslant 1} E_{i}^{\omega, l} a^{*}\left(\phi_{i}\right) a\left(\phi_{i}\right),
$$

where $a^{*}\left(\phi_{i}\right), a\left(\phi_{i}\right)$ are the creation and annihilation operators (satisfying the boson Canonical Commutation Relations) in the one-particle eigenstates $\left\{\phi_{i}:=\phi_{i}^{\omega, l}\right\}_{i} \geqslant 1$ of $h_{l}^{\omega}$. Then, the grandcanonical Hamiltonian of the perfect Bose gas in a random external potential is given by:

$$
H_{l}(\mu):=H_{l}-\mu N_{l}=\sum_{i \geqslant 1}\left(E_{i}^{\omega, l}-\mu\right) N_{l}\left(\phi_{i}\right)
$$

where $N_{l}\left(\phi_{i}\right):=a^{*}\left(\phi_{i}\right) a\left(\phi_{i}\right)$ is the operator for the number of particles in the eigenstate $\phi_{i}$, $N_{l}:=\sum_{i} N_{l}\left(\phi_{i}\right)$ is the operator for the total number of particles in $\Lambda_{l}$ and $\mu$ is the chemical 
potential. Note that $N_{l}$ can be expanded over any basis in the space $\mathscr{H}_{l}$, and in particular over the one defined by the free one-particle kinetic-energy eigenstates $\left\{\psi_{k}^{l}, \varepsilon_{k}\right\}_{k}$.

We denote by $\langle-\rangle_{l}$ the equilibrium state defined by the Hamiltonian $H_{l}(\mu)$ :

$$
\langle A\rangle_{l}(\beta, \mu):=\frac{\operatorname{Tr}_{\mathscr{F}_{2}}\left\{\exp \left(-\beta H_{l}(\mu)\right) A\right\}}{\operatorname{Tr}_{\mathscr{F}_{l}} \exp \left(-\beta H_{l}(\mu)\right)} .
$$

For simplicity, we shall omit in the following the explicit mention of the dependence on the thermodynamic parameters $(\beta, \mu)$. Finally, we define the Thermodynamic Limit (TL) as the limit, when $l \rightarrow \infty$.

\section{Generalized BEC in one-particle random eigenstates}

In this section we consider the possibility of macroscopic occupation of the one-particle random Schrödinger operator (2.3) eigenstates $\left\{\phi_{i}\right\}_{i \geqslant 1}$. Recall that the corresponding limiting IDS, $\nu(E)$, is defined as:

$$
\nu(E):=\lim _{l \rightarrow \infty} \nu_{l}^{\omega}(E)=\lim _{l \rightarrow \infty} \frac{1}{V_{l}} \sharp\left\{i: E_{i}^{\omega, l} \leqslant E\right\} .
$$

Although the finite-volume IDS, $\nu_{l}^{\omega}(E)$, are random measures, one can check that for homogeneous ergodic random potentials the limit (3.1) has the property of self-averaging [12]. This means that $\nu(E)$ is almost surely (a. s.) a non-random measure. Let us define a (random) particle density occupation measures $m_{l}$ by:

$$
m_{l}(A):=\frac{1}{V_{l}} \sum_{i: E_{i}^{w, l} \in A}\left\langle N_{l}\left(\phi_{i}\right)\right\rangle_{l}, \quad A \subset \mathbb{R} .
$$

Then using standard methods, one can prove that this sequence of measures has (a. s.) a nonrandom weak-limit $m$, see (3.8) below. Moreover, if the critical density

$$
\rho_{\mathrm{c}}:=\lim _{\mu \rightarrow 0} \int_{0}^{\infty} \frac{1}{\mathrm{e}^{\beta(E-\mu)}-1} \nu(\mathrm{d} E)
$$

is finite, then one obtains a generalized Bose-Einstein condensation (g-BEC) in the sense that this measure $m$ has an atom at the bottom of the spectrum of the random Schrödinger operator, which by (iii), section 2 , is assumed to be at 0 :

$$
m(\{0\})=\lim _{\delta \downarrow 0} \lim _{l \rightarrow \infty} \sum_{i: E_{i}^{w, l} \leqslant \delta} \frac{1}{V_{l}}\left\langle N_{l}\left(\phi_{i}\right)\right\rangle_{l}= \begin{cases}0 & \text { if } \bar{\rho}<\rho_{\mathrm{c}} \\ \bar{\rho}-\rho_{\mathrm{c}} & \text { if } \bar{\rho} \geqslant \rho_{\mathrm{c}}\end{cases}
$$

where $\bar{\rho}$ denotes a (fixed) mean density [4,6]. Physically, this corresponds to the macroscopic occupation of the set of eigenstates $\phi_{i}$ with energy close to the ground state $\phi_{1}$. However, we have to stress that BEC in this sense does not necessarily imply a macroscopic occupation of the ground state. In fact, the condensate can be spread over many (and even infinitely many) states.

These various situations correspond to classification of the g-BEC on the types I, II and III, introduced in the eighties by van den Berg-Lewis-Pulé, see e. g. [13] or $[7,14]$. The most striking case is type III when generalized BEC occurs in the sense of equation (3.4) even though none of the eigenstates $\phi_{i}$ is macroscopically occupied. The realization of different types depends on how the relative gaps between the eigenvalues $E_{i}$ at the bottom of the spectrum vanish in the TL. To our knowledge, analysis of this behaviour in random system has only been realised in some particular cases, see [6] for a comprehensive presentation. The concept of generalized BEC is more stable than the standard one-mode BEC, since it depends on the global low-energy behaviour of the density of states, especially on its ability to make the critical density (3.3) finite. We also note that, since the IDS (3.1) is not random, the same it true for the amount of the g-BEC (3.4). 
We can also obtain an explicit expression for the limiting measure $m$. Note that we have fixed the mean density $\bar{\rho}$, which implies that we require the chemical potential $\mu$ to satisfy the equation:

$$
\bar{\rho}=\left\langle N_{l}\right\rangle_{l}(\beta, \mu)=\frac{1}{V_{l}} \sum_{i \geqslant 1} \frac{1}{\mathrm{e}^{\beta\left(E_{i}^{\omega, l}-\mu\right)}-1},
$$

for any $l$. Since the system is disordered, the unique solution $\mu_{l}^{\omega}:=\mu_{l}^{\omega}(\beta, \bar{\rho})$ of this equation is a random variable, which is a. s. non-random in the TL $[4,6]$. In the rest of this paper we denote the non-random $\mu_{\infty}:=$ a. s.- $\lim _{l \rightarrow \infty} \mu_{l}^{\omega}$. By condition (iii), section 2 , and by (3.7) it is a continuous function of $\bar{\rho}$ :

$$
\mu_{\infty}(\beta, \bar{\rho})= \begin{cases}0 & \text { if } \bar{\rho} \geqslant \rho_{\mathrm{c}} \\ \bar{\mu}<0 & \text { if } \bar{\rho}<\rho_{\mathrm{c}}\end{cases}
$$

where $\bar{\mu}:=\bar{\mu}(\beta, \bar{\rho})$ is a (unique) solution of the equation:

$$
\bar{\rho}=\int_{0}^{\infty} \frac{1}{\mathrm{e}^{\beta(E-\mu)}-1} \nu(\mathrm{d} E)
$$

for $\bar{\rho} \leqslant \rho_{\mathrm{c}}$.

Remark 3.1 Note that $\mu_{\infty}$ is non-positive (3.6), which is not true in general for the random finitevolume solution $\mu_{l}^{\omega}$. Indeed, the only restriction we have is that $\mu_{l}^{\omega}<E_{1}^{\omega, l}$, which is the well-known condition for the pressure of the perfect Bose gas to exist. We return to this question in section 4 for the case of random BEC in the free one-particle kinetic-energy operator eigenfunctions.

We also recall that for (3.6) the explicit expression of the weak limit for the general particle density occupation measure is:

$$
m(\mathrm{~d} E)= \begin{cases}\left(\bar{\rho}-\rho_{\mathrm{c}}\right) \delta_{0}(\mathrm{~d} E)+\left(\mathrm{e}^{\beta E}-1\right)^{-1} \nu(\mathrm{d} E) & \text { if } \bar{\rho} \geqslant \rho_{\mathrm{c}} \\ \left(\mathrm{e}^{\beta\left(E-\mu_{\infty}\right)}-1\right)^{-1} \nu(\mathrm{d} E) & \text { if } \bar{\rho}<\rho_{\mathrm{c}}\end{cases}
$$

We end this section with a comment about the difference between the model of the perfect Bose gas embedded into a random potential and the free Bose gas. In the latter case, one should consider the IDS of the one-particle kinetic-energy operator (2.1), which is given by the Weyl formula:

$$
\nu^{0}(E)=C_{d} E^{d / 2}
$$

where is $C_{d}$ is a constant term depending only on the dimensionality $d$. It is known that for this IDS, the critical density (3.3) is finite only when $d>2$, and hence the fact that BEC does not occur for low dimensions. On the other hand, a common feature of Schrödinger operators with regular, stationary, non-negative ergodic random potentials is the so-called Lifshitz tails behaviour of the IDS near the bottom of the spectrum. If the lower edge of the spectrum coincides with $E=0$ (condition (iii), section 2), this means that for the IDS one has the "doublelogarithmic" asymptote, see [12]:

$$
\lim _{E \rightarrow 0^{+}} \frac{\ln |\ln (\nu(E))|}{\ln E}=-\frac{d}{2} .
$$

Here we consider a stronger form of this asymptotic behaviour, namely:

$$
\lim _{E \rightarrow 0^{+}}\left(-E^{d / 2}\right) \ln (\nu(E)) \geqslant a>0
$$

for some constant $a$. The technical conditions assumed throughout this paper are sufficient for (3.11) to hold. Lifshitz tails in this form are in particular true in the case of Poisson random potentials. In general, however, there can be a logarithmic correction to (3.11), so that one can only expect the weak form (3.10). Hence, the critical density (3.3) is finite in any dimension, and therefore enhances BEC in the sense of (3.4) even for $d=1,2$. This was shown in [4,6], where some specific examples of one-dimensional Poisson disordered systems exhibiting g-BEC in the sense of (3.4) were studied. 


\section{Generalized BEC in one-particle kinetic energy eigenstates}

\subsection{Occupation measure for one-particle kinetic energy eigenstates}

Similar to (3.2), we introduce the sequence of particle occupation measure $\tilde{m}_{l}$ for kinetic energy eigenfunctions $\left\{\psi_{k}:=\psi_{k}^{l}\right\}_{k \in \Lambda_{l}^{*}}$ :

$$
\tilde{m}_{l}(A):=\frac{1}{V_{l}} \sum_{k: \varepsilon_{k} \in A}\left\langle N_{l}\left(\psi_{k}\right)\right\rangle_{l}, \quad A \subset \mathbb{R},
$$

but now in the random equilibrium states $\langle-\rangle_{l}$ corresponding to the perfect boson gas with Hamiltonian (2.5).

Note that, contrary to the last section, the standard arguments used to prove the existence of a limiting measure in TL are not valid for $\tilde{m}_{l}$, since the kinetic energy operator (2.1) and the random Schrödinger operator (2.3) do not commute.

We also remark that even if we know that the measure $m$ (3.8) has an atom at the edge of the spectrum (g-BEC), we cannot deduce that the limiting measure $\tilde{m}$ (assuming that it exists) also manifests g-BEC in the free kinetic energy eigenstates $\psi_{k}$.

Our motivation to study this problem is that in view of the well-known Bogoliubov approximation, it is the latter that is required, see [7-9]. Indeed, let the second-quantized form of the interaction term be expressed in terms of creation/annihilation operators in states $\psi_{k}^{l}$, eigenfunctions of kinetic-energy operator (2.1). Then the so-called first Bogoliubov approximation (Bogoliubov ansatz) assumes that only terms involving creation and/or annihilation operators of particles in the ground state $\psi_{1}^{l}$ are relevant. The Bogoliubov theory is nontrivial if there is macroscopic occupation of this zero-mode kinetic-energy operator ground-state. Therefore, the g-BEC in the sense (3.4) is not sufficient to apply the Bogoliubov ansatz.

Now we formulate the main result of this section. Let

$$
\Omega_{\left(x, x^{\prime}\right)}^{T}:=\left\{\xi: \xi(0)=x, \xi(T)=x^{\prime}\right\}
$$

be the set of continuous trajectories (paths) $\{\xi(s)\}_{s=0}^{T}$ in $\mathbb{R}^{d}$, connecting the points $x, x^{\prime}$, and let $w^{T}$ denote the normalized Wiener measure on this set.

Theorem 4.1 The sequence of measures $\tilde{m}_{l}$ converges a. s. in a weak sense to a non-random measure $\tilde{m}$, which is given by:

$$
\tilde{m}(\mathrm{~d} \varepsilon)= \begin{cases}\left(\bar{\rho}-\rho_{\mathrm{c}}\right) \delta_{0}(\mathrm{~d} \varepsilon)+F(\varepsilon) \mathrm{d} \varepsilon & \text { if } \bar{\rho} \geqslant \rho_{\mathrm{c}} \\ F(\varepsilon) \mathrm{d} \varepsilon & \text { if } \bar{\rho}<\rho_{\mathrm{c}}\end{cases}
$$

with density $F(\varepsilon)$ defined by:

$$
F(\varepsilon)=(2 \varepsilon)^{d / 2-1} \int_{S_{d}^{1}} \mathrm{~d} \sigma g\left(\sqrt{2 \varepsilon} n_{\sigma}\right)
$$

Here, $S_{d}^{1}$ denotes the unit sphere in $\mathbb{R}^{d}$ centered at the origin, $n_{\sigma}$ the unit outward drawn normal vector, and $\mathrm{d} \sigma$ the surface measure of $S_{d}^{1}$. The function $g$ is as follows

$$
g(k)=\frac{1}{(2 \pi)^{d / 2}} \int_{\mathbb{R}^{d}} \mathrm{~d} x \mathrm{e}^{\mathrm{i} k x} \sum_{n \geqslant 1} \mathrm{e}^{n \beta \mu_{\infty}} \frac{\mathrm{e}^{-\|x\|^{2}(1 / 2 n \beta)}}{(2 \pi n \beta)^{d / 2}} \mathbb{E}_{\omega}\left\{\int_{\Omega_{(0, x)}^{n \beta}} w^{n \beta}(\mathrm{d} \xi) \mathrm{e}^{-\int_{0}^{n \beta} \mathrm{d} s v^{\omega}(\xi(s))}\right\},
$$

with expectation $\mathbb{E}_{\omega}$ on the probability space $(\Omega, \mathcal{F}, \mathbb{P})$.

Note that since the Wiener measures $w^{n \beta}$ on $\Omega_{(0, x)}^{n \beta}$ are normalized, we recover from (4.2) the expression for the free Bose gas if we put $v^{\omega}=0$.

Before proceeding with the proof, we give some comments about these results. 
(a) First, the existence of a non-trivial limiting kinetic energy states occupation measure provides a rigorous basis for discussing the macroscopic occupation of the free Bose gas eigenstates.

(b) Moreover, both occupation measures (3.8) and (4.1) do not only exhibit simultaneously an atom at the bottom of the spectrum, but these atoms have the same non-random weights. It is quite surprising that the generalized BEC triggered by the Lifshitz tail in a low dimension disordered system produces the same value of the generalized BEC in the lowest free kinetic energy states.

\subsection{Proofs}

We start by expanding the measure $\tilde{m}$ in terms of the random equilibrium mean-values of occupation numbers in the corresponding eigenstates $\phi_{i}$. Using the linearity (respectively conjugate linearity) of the creation and annihilation operators one obtains:

$$
\begin{aligned}
\tilde{m}_{l}(A) & =\frac{1}{V_{l}} \sum_{k: \varepsilon_{k} \in A}\left\langle a^{*}\left(\psi_{k}\right) a\left(\psi_{k}\right)\right\rangle_{l} \\
& =\frac{1}{V_{l}} \sum_{i, j} \sum_{k: \varepsilon_{k} \in A} \overline{\left(\phi_{i}, \psi_{k}\right)}\left(\phi_{i},\left(\psi_{k}\right)\left\langle a^{*}\left(\phi_{i}\right) a\left(\phi_{j}\right)\right\rangle_{l}\right. \\
& =\frac{1}{V_{l}} \sum_{i} \sum_{k: \varepsilon_{k} \in A}\left|\left(\phi_{i}, \psi_{k}\right)\right|^{2}\left\langle a^{*}\left(\phi_{i}\right) a\left(\phi_{i}\right)\right\rangle_{l} .
\end{aligned}
$$

In the last equality, we have used the fact that $\left[H_{l}(\mu), N_{l}\left(\phi_{i}\right)\right]=0$ for all $i$, which implies that:

$$
\left\langle a^{*}\left(\phi_{i}\right) a\left(\phi_{j}\right)\right\rangle_{l}=0 \quad \text { if } \quad i \neq j
$$

This is the analogue of the momentum conservation law in the free Bose gas. Although, it has a different physical sense: the conservation of the particle number in each random eigenstate $\phi_{i}$.

We first prove two important lemmas. In neither of them we shall assume that the sequence $\tilde{m}_{l}$ has a weak limit, instead we consider only some convergent subsequence. Note that at least one such subsequence always exists, see [17].

The first result states that if there is condensation in the lowest random eigenstates $\left\{\phi_{i}\right\}_{i}$, then there is also condensation in the lowest kinetic-energy states $\left\{\psi_{k}\right\}_{k}$. Moreover, the amount of the latter condensate density has to be not less than the former.

Lemma 4.1 Let $\left\{\tilde{m}_{l_{r}}\right\}_{r \geqslant 1}$ be a convergent subsequence. We denote by $\tilde{m}$ its (weak) limit. Then:

$$
\tilde{m}(\{0\}) \geqslant m(\{0\})= \begin{cases}\bar{\rho}-\rho_{\mathrm{c}} & \text { if } \bar{\rho} \geqslant \rho_{\mathrm{c}} \\ 0 & \text { if } \bar{\rho}<\rho_{\mathrm{c}}\end{cases}
$$

Proof: Let $\gamma>0$. Using the expansion of the functions $\psi_{k}$ in the basis $\left\{\phi_{i}\right\}_{i \geqslant 1}$, we obtain:

$$
\begin{aligned}
\tilde{m}([0, \gamma]) & =\lim _{r \rightarrow \infty} \frac{1}{V_{l_{r}}} \sum_{k: \varepsilon_{k} \leqslant \gamma}\left\langle N_{l_{r}}\left(\psi_{k}\right)\right\rangle_{l_{r}} \\
& =\lim _{r \rightarrow \infty} \frac{1}{V_{l_{r}}} \sum_{k: \varepsilon_{k} \leqslant \gamma} \sum_{i \geqslant 1}\left|\left(\phi_{i}, \psi_{k}\right)\right|^{2}\left\langle N_{l_{r}}\left(\phi_{i}\right)\right\rangle_{l_{r}} \\
& \geqslant \lim _{r \rightarrow \infty} \frac{1}{V_{l_{r}}} \sum_{k: \varepsilon_{k} \leqslant \gamma} \sum_{i: E_{i} \leqslant \delta}\left|\left(\phi_{i}, \psi_{k}\right)\right|^{2}\left\langle N_{l_{r}}\left(\phi_{i}\right)\right\rangle_{l_{r}}
\end{aligned}
$$

for any $\delta>0$. The non-negativity of the random potential (2.4) implies:

$$
\sum_{k: \varepsilon_{k}>\gamma}\left|\left(\phi_{i}, \psi_{k}\right)\right|^{2} \leqslant \sum_{k: \varepsilon_{k}>\gamma} \frac{\varepsilon_{k}}{\gamma}\left|\left(\phi_{i}, \psi_{k}\right)\right|^{2} \leqslant \frac{1}{\gamma} \sum_{k \geqslant 1} \varepsilon_{k}\left|\left(\phi_{i}, \psi_{k}\right)\right|^{2}=\frac{1}{\gamma}\left(\phi_{i}, h_{l}^{0} \phi_{i}\right) \leqslant \frac{1}{\gamma}\left(\phi_{i}, h_{l}^{\omega} \phi_{i}\right)=\frac{E_{i}^{\omega}}{\gamma}
$$


We then obtain:

$$
\begin{aligned}
\tilde{m}([0, \gamma]) & \geqslant \lim _{r \rightarrow \infty} \frac{1}{V_{l_{r}}} \sum_{i: E_{i} \leqslant \delta}\left\langle N_{l_{r}}\left(\phi_{i}\right)\right\rangle_{l_{r}}\left(1-\sum_{k: \varepsilon_{k}>\gamma}\left|\left(\phi_{i}, \psi_{k}\right)\right|^{2}\right) \\
& \geqslant \lim _{r \rightarrow \infty} \frac{1}{V_{l_{r}}} \sum_{i: E_{i} \leqslant \delta}\left\langle N_{l_{r}}\left(\phi_{i}\right)\right\rangle_{l_{r}}\left(1-E_{i} / \gamma\right) \\
& \geqslant \lim _{r \rightarrow \infty}(1-\delta / \gamma) \frac{1}{V_{l_{r}}} \sum_{i: E_{i} \leqslant \delta}\left\langle N_{l_{r}}\left(\phi_{i}\right)\right\rangle_{l_{r}}=(1-\delta / \gamma) m([0, \delta]) \geqslant 0 .
\end{aligned}
$$

But $\delta$ is arbitrary, and the lemma follows by letting $\delta \rightarrow 0$.

In the next lemma, we show that the measure $\tilde{m}(4.1)$ can have an atom only at zero kinetic energy.

Lemma 4.2 Let $\left\{\tilde{m}_{l_{r}}\right\}_{r} \geqslant 1$ be a convergent subsequence, and $\tilde{m}$ be its (weak) limit. Then, it is absolutely continuous on $\mathbb{R}_{+}:=(0, \infty)$.

Proof: Let $A$ be a Borel subset of $(0, \infty)$, with Lebesgue measure 0 , and let $a$ be such that $\inf A>a>0$. Then:

$$
\begin{aligned}
\tilde{m}_{l_{r}}(A) & =\frac{1}{V_{l_{r}}} \sum_{k: \varepsilon_{k} \in A}\left\langle N_{l_{r}}\left(\psi_{k}\right)\right\rangle_{l_{r}} \\
& =\frac{1}{V_{l_{r}}} \sum_{k: \varepsilon_{k} \in A} \sum_{i}\left|\left(\phi_{i}, \psi_{k}\right)\right|^{2}\left\langle N_{l_{r}}\left(\phi_{i}\right)\right\rangle_{l_{r}} \\
& =\frac{1}{V_{l_{r}}} \sum_{k: \varepsilon_{k} \in A} \sum_{i: E_{i} \leqslant \alpha}\left|\left(\phi_{i}, \psi_{k}\right)\right|^{2}\left\langle N_{l_{r}}\left(\phi_{i}\right)\right\rangle_{l_{r}}+\frac{1}{V_{l_{r}}} \sum_{k: \varepsilon_{k} \in A} \sum_{i: E_{i}>\alpha}\left|\left(\phi_{i}, \psi_{k}\right)\right|^{2}\left\langle N_{l_{r}}\left(\phi_{i}\right)\right\rangle_{l_{r}}(
\end{aligned}
$$

for some $\alpha>0$. Next, we use (2.4) to get the following estimate:

$$
E_{i}=\left(\phi_{i}, h_{l}^{\omega} \phi_{i}\right) \geqslant\left(\phi_{i}, h_{l}^{0} \phi_{i}\right)=\sum_{k} \varepsilon_{k}\left|\left(\phi_{i}, \psi_{k}\right)\right|^{2} \geqslant a \sum_{k: \varepsilon_{k} \in A}\left|\left(\phi_{i}, \psi_{k}\right)\right|^{2} .
$$

Since the equilibrium value of the occupation numbers $\left\langle N_{l}\left(\phi_{i}\right)\right\rangle_{l}=\left\{\mathrm{e}^{E_{i}-\mu}-1\right\}^{-1}$ are decreasing with $i$, the estimate (4.4) implies:

$$
\tilde{m}_{l_{r}}(A) \leqslant \frac{1}{V_{l_{r}}} \frac{1}{a} \sum_{i: E_{i} \leqslant \alpha} E_{i}\left\langle N_{l_{r}}\left(\phi_{i}\right)\right\rangle_{l_{r}}+\left\langle N_{l_{r}}\left(\phi_{i_{\alpha}}\right)\right\rangle_{l_{r}} \frac{1}{V_{l_{r}}} \sum_{k: \varepsilon_{k} \in A} 1,
$$

where $\phi_{i_{\alpha}}$ denotes the eigenstate of $h_{l}^{\omega}$ with the smallest eigenvalue greater than $\alpha$. Using again the monotonousness and the finite-volume IDS (3.1) we can get an upper bound for the mean occupation number in the second term of (4.5), since:

$$
\bar{\rho}=\frac{1}{V_{l}} \sum_{i}\left\langle N_{l}\left(\phi_{i}\right)\right\rangle_{l} \geqslant \frac{1}{V_{l}} \sum_{i: E_{i} \leqslant \alpha}\left\langle N_{l}\left(\phi_{i}\right)\right\rangle_{l} \geqslant\left\langle N_{l}\left(\phi_{i_{\alpha}}\right)\right\rangle_{l} \nu_{l}^{\omega}(\alpha) .
$$

Combining (4.5) and (4.6) we obtain:

$$
\tilde{m}_{l_{r}}(A) \leqslant \frac{\alpha \bar{\rho}}{a}+\frac{\bar{\rho}}{\nu_{l_{r}}^{\omega}(\alpha)} \int_{A} \nu_{l_{r}}^{0}(\mathrm{~d} \varepsilon) .
$$

Since the measure $\nu^{0}(3.9)$ is absolutely continuous with respect to the Lebesgue measure, and $\nu(\alpha)$ is strictly positive for any $\alpha>0$ the limit $r \rightarrow \infty$ in (4.7) gives:

$$
\tilde{m}(A) \leqslant \frac{\alpha \bar{\rho}}{a} .
$$

But $\alpha>0$ can be chosen arbitrarily small and thus $\tilde{m}(A)=0$. To finish the proof, note that any Borel subset of $(0, \infty)$ can be expressed as a countable union of disjoint subsets with non-zero infimum. Our arguments then can be applied to each of them. 
Remark 4.1 Lemmas 4.1 and 4.2 are fairly general, since they require only the non-negativity of the potential and in the random case, ergodicity. In particular, they apply to non-random (weak) external potentials, that we consider below, as well as to some models of an interacting Bose gas, as long as the many-particle Hamiltonian still satisfies the commutation relation $\left[H_{l}(\mu), N_{l}\left(\phi_{i}\right)\right]=0$. In particular this holds in the case of models with interactions, which are diagonal in the occupation number operators.

Above we exploited the fact that the sequence $\left\{\tilde{m}_{l}\right\}_{l \geqslant 1}$ has at least one accumulation point. However, to prove the convergence, we need to make use of some particular and explicit features of the perfect Bose gas, as well as a more detailed information about the properties of the external (random) potential. In particular, we shall need some estimates of the (random) finite-volume integrated density of states.

\section{On the nature of the generalized condensates in the Luttinger-Sy model}

In this section, we study the van den Berg-Lewis-Pulé classification of generalized BE condensation (see discussion in section 3) in a particular case of the so-called Luttinger-Sy model with point impurities [3].

Let $u(x) \geqslant 0, x \in \mathbb{R}$, be a continuous function with a compact support called a (repulsive) single-impurity potential. Let $\left\{\mu_{\lambda}^{\omega}\right\}_{\omega \in \Omega}$ be the random Poisson measure on $\mathbb{R}$ with intensity $\lambda>0$ :

$$
\mathbb{P}\left(\left\{\omega \in \Omega: \mu_{\lambda}^{\omega}(\Lambda)=n\right\}\right)=\frac{(\lambda|\Lambda|)^{n}}{n !} \mathrm{e}^{-\lambda|\Lambda|}, \quad n \in \mathbb{N}_{0}=\mathbb{N} \cup\{0\},
$$

for any bounded Borel set $\Lambda \subset \mathbb{R}$. Then the non-negative random potential $v^{\omega}$ generated by the Poisson distributed local impurities has realizations

$$
v^{\omega}(x):=\int_{\mathbb{R}} \mu_{\lambda}^{\omega}(\mathrm{d} y) u(x-y)=\sum_{x_{j}^{\omega} \in X^{\omega}} u\left(x-x_{j}^{\omega}\right) .
$$

Here the random set $X^{\omega}$ corresponds to impurity positions $X^{\omega}=\left\{x_{j}^{\omega}\right\}_{j} \subset \mathbb{R}$, which are the atoms of the random point Poisson measure, i. e., $\sharp\left\{X^{\omega}\lceil\Lambda\}=\mu_{\lambda}^{\omega}(\Lambda)\right.$ is the number of impurities in the set $\Lambda$. Since the expectation $\mathbb{E}\left(\mu_{\lambda}^{\omega}(\Lambda)\right)=\lambda|\Lambda|$, the parameter $\lambda$ coincides with the density of impurities on $\mathbb{R}$.

Luttinger and Sy defined their model by restricting the single-impurity potential to the case of point $\delta$-potential with amplitude $a \rightarrow+\infty$. Then, the corresponding random potential (5.2) takes the form:

$$
v_{a}^{\omega}(x):=\int_{\mathbb{R}} \mu_{\lambda}^{\omega}(\mathrm{d} y) a \delta(x-y)=a \sum_{x_{j}^{\omega} \in X^{\omega}} \delta\left(x-x_{j}^{\omega}\right) .
$$

Now the self-adjoint one-particle random Schrödinger operator $h_{a}^{\omega}:=h^{0} \dot{+} v_{a}^{\omega}$ is defined in the sense of the sum of quadratic forms (2.2). The strong resolvent limit $h_{\mathrm{LS}}^{\omega}:=s . r . \lim _{a \rightarrow+\infty} h_{a}^{\omega}$ is the Luttinger-Sy model.

Since $X^{\omega}$ generates a set of intervals $\left\{I_{j}^{\omega}:=\left(x_{j-1}^{\omega}, x_{j}^{\omega}\right)\right\}_{j}$ of lengths $\left\{L_{j}^{\omega}:=x_{j}^{\omega}-x_{j-1}^{\omega}\right\}_{j}$, one gets decompositions of the one-particle Luttinger-Sy Hamiltonian:

$$
h_{\mathrm{LS}}^{\omega}=\bigoplus_{j} h_{D}\left(I_{j}^{\omega}\right), \quad \operatorname{dom}\left(h_{\mathrm{LS}}^{\omega}\right) \subset \bigoplus_{j} L^{2}\left(I_{j}^{\omega}\right), \quad \omega \in \Omega,
$$

into random disjoint free Schrödinger operators $\left\{h_{\mathrm{D}}\left(I_{j}^{\omega}\right)\right\}_{j, \omega}$ with Dirichlet boundary conditions at the end-points of intervals $\left\{I_{j}^{\omega}\right\}_{j}$. Then the Dirichlet restriction $h_{l, \mathrm{D}}^{\omega}$ of the Hamiltonian $h_{\mathrm{LS}}^{\omega}$ to a fixed interval $\Lambda_{l}=(-l / 2, l / 2)$ and the corresponding change of notations are evident: e. g., $\left\{I_{j}^{\omega}\right\}_{j} \mapsto\left\{I_{j}^{\omega}\right\}_{j=1}^{M^{l}(\omega)}$, where $M^{l}(\omega)$ is total number of subintervals in $\Lambda_{l}$ corresponding to the set $X^{\omega}$. For rigorous definitions and some results concerning this model we refer the reader to [6]. 
Since this particular choice of random potential is capable of producing Lifshitz tails in the sense of (3.11), see Proposition 3.2 in [6], it follows that such a model exhibits a generalized BEC in random eigenstates, see (3.4). In fact, it was shown in [6] that only the random ground state $\phi_{1}^{\omega, l}$ of $h_{l, \mathrm{D}}^{\omega}$ is macroscopically occupied. In our notations this means that

$$
\begin{aligned}
& \lim _{l \rightarrow \infty} \frac{1}{l}\left\langle N_{l}\left(\phi_{1}^{\omega, l}\right)\right\rangle_{l}= \begin{cases}0 & \text { if } \bar{\rho}<\rho_{\mathrm{c}}, \\
\bar{\rho}-\rho_{\mathrm{c}} & \text { if } \bar{\rho} \geqslant \rho_{\mathrm{c}},\end{cases} \\
& \lim _{l \rightarrow \infty} \frac{1}{l}\left\langle N_{l}\left(\phi_{i}^{\omega, l}\right)\right\rangle_{l}=0, \quad \text { for all } i>1 .
\end{aligned}
$$

According to the van den Berg-Lewis-Pulé classification this corresponds to the type $I$ Bosecondensation in the random eigenstates $\left\{\phi_{i}^{\omega}\right\}_{i \geqslant 1}$.

Following the line of reasoning of section 4, we now consider the corresponding BEC in the kinetic-energy eigenstates. We retain the notation used in that section and briefly explain the minor changes required in applying our method to the Luttinger-Sy model.

We first state the equivalent of Theorem 4.1 for this particular model.

Theorem 5.1 Theorem 4.1 holds with the function $g$ defined as follows

$$
\begin{aligned}
g(k)= & \frac{1}{(2 \pi)^{d / 2}} \int_{\mathbb{R}^{d}} \mathrm{~d} x \mathrm{e}^{\mathrm{i} k x} \sum_{n \geqslant 1} \mathrm{e}^{n \beta \mu_{\infty}} \frac{\mathrm{e}^{-\|x\|^{2}(1 / 2 n \beta)}}{(2 \pi n \beta)^{d / 2}} \\
& \times \int_{\Omega_{(0, x)}^{n \beta}} w^{n \beta}(\mathrm{d} \xi) \exp \left(-\lambda\left(\sup _{s} \xi(s)-\inf _{s} \xi(s)\right)\right) .
\end{aligned}
$$

The scheme of the proof is the same as the above, cf. section 4. First, we note that Lemmas 4.1 and 4.2 apply immediately. The positivity of the random potential has to be understood in terms of quadratic forms, see (2.4).

First, suppose that there is at least one impurity in the box, then the eigenvalues will be of the form (for some $j$ )

$$
\left(n^{2} \pi^{2}\right) /\left(L_{j}^{\omega}\right)^{2}, \quad n=1,2, \ldots
$$

if $I_{j}^{\omega}$ is an inner interval (that is, its two endpoints correspond to impurities), and

$$
\left((n+1 / 2)^{2} \pi^{2}\right) /\left(L_{j}^{\omega}\right)^{2}, \quad n=0,1,2, \ldots
$$

if $I_{j}^{\omega}$ is an outer interval (that is, one endpoint corresponds to an impurity, and the other one to the boundary of $\Lambda_{l}$ ). Therefore, $E_{1}^{\omega, l, N} \geqslant B / l^{2}$ since obviously $L_{j}^{\omega}<l$. Now, if there is no impurity in the box $\Lambda_{l}$, then $E_{1}^{\omega, l, N}=0<B / l^{2}$. But due to the Poisson distribution (5.1) this happens with probability $\mathrm{e}^{-\lambda l}$.

Our next step is to split the measure $\tilde{m}_{l}$ into two, $\tilde{m}_{l}^{(1)}$ and $\tilde{m}_{l}^{(2)}$, and to prove the following statement:

Theorem 5.2 For any $d \geqslant 1$, the sequence of Laplace transforms of the measures $\tilde{m}_{l}^{(1)}$ :

$$
f_{l}\left(t ; \beta, \mu_{l}\right):=\int_{\mathbb{R}} \tilde{m}_{l}^{(1)}(\mathrm{d} \varepsilon) \mathrm{e}^{-t \varepsilon}
$$

converges for any $t>0$ to a (non-random) limit $f\left(t ; \beta, \mu_{\infty}\right)$, which is given by:

$$
\begin{aligned}
f\left(t ; \beta, \mu_{\infty}\right) & =\sum_{n \geqslant 1} \mathrm{e}^{n \beta \mu_{\infty}} \int_{\mathbb{R}^{d}} \mathrm{~d} x \frac{\mathrm{e}^{-\|x\|^{2}(1 / 2 n \beta+1 / 2 t)}}{\left(4 \pi^{2} \operatorname{tn} \beta\right)^{d / 2}} \\
& \times \int_{\Omega_{(0, x)}^{n \beta}} w^{n \beta}(\mathrm{d} \xi) \exp \left(-\lambda\left(\sup _{s} \xi(s)-\inf _{s} \xi(s)\right)\right) .
\end{aligned}
$$


Proof: We can use the ergodic theorem to obtain:

$$
\lim _{l \rightarrow \infty} a_{l}(n)=\mathrm{e}^{n \beta \mu_{\infty}} \mathbb{E}_{\omega} \int_{\mathbb{R}} \mathrm{d} x \frac{\mathrm{e}^{-\|x\|^{2}(1 / 2 n \beta+1 / 2 t)}}{\left(4 \pi^{2} \operatorname{tn} \beta\right)^{d / 2}} \sum_{j} \int_{\Omega_{(0, x)}^{n \beta}} w^{n \beta}(\mathrm{d} \xi) \chi_{I_{j}{ }^{\omega}, n \beta}(\xi) .
$$

We have used the fact that the Dirichlet boundary conditions at the impurities split up the space $\mathcal{H}_{l}$ into a direct sum of Hilbert spaces (see (5.4)). This can be seen from the expression

$$
\lim _{l \rightarrow \infty} a_{l}(n)=\mathrm{e}^{n \beta \mu_{\infty}} \int_{\mathbb{R}} \mathrm{d} x \frac{\mathrm{e}^{-\|x\|^{2}(1 / 2 n \beta+1 / 2 t)}}{\left(4 \pi^{2} \operatorname{tn} \beta\right)^{d / 2}} \mathbb{E}_{\omega} \int_{\Omega_{(0, x)}^{n \beta}} w^{n \beta}(\mathrm{d} \xi) \mathrm{e}^{-\int_{0}^{n \beta} \mathrm{d} s a \sum_{x_{j}^{\omega} \in X \omega} \delta\left(\xi(s)-x_{j}^{\omega}\right)}
$$

by formally putting the amplitude, $a$, of the point impurities (5.3) equal to $+\infty$. Because of the characteristic functions $\chi_{I_{j}^{\omega}, n \beta}$, which constrain the paths $\xi$ to remain in the interval $I_{j}^{\omega}$ in time $n \beta$, the sum in (5.6) reduces to only one term:

$$
\lim _{l \rightarrow \infty} a_{l}(n)=\mathrm{e}^{n \beta \mu_{\infty}} \int_{\mathbb{R}} \mathrm{d} x \frac{\mathrm{e}^{-\|x\|^{2}(1 / 2 n \beta+1 / 2 t)}}{\left(4 \pi^{2} \operatorname{tn} \beta\right)^{d / 2}} \mathbb{E}_{\omega} \int_{\Omega_{(0, x)}^{n \beta}} w^{n \beta}(\mathrm{d} \xi) \chi_{\left(a_{\omega}, b_{\omega}\right), n \beta}(\xi),
$$

where $\left(a_{\omega}, b_{\omega}\right)$, is the interval among the $I_{j}^{\omega}$ 's which contains 0 .

The expression in (5.7) can be further simplified by computing the expectation $\mathbb{E}_{\omega}$ explicitly. First, note that the Poisson impurity positions: $a_{\omega}, b_{\omega}$ are independent random variables and by definition, $a_{\omega}$ is negative while $b_{\omega}$ is positive. For the random variable $b_{\omega}$ the distribution function is:

$$
\mathbb{P}\left(b_{\omega}<b\right):=\mathbb{P}\{(0, b) \text { contains at least one impurity }\}=1-\mathrm{e}^{-\lambda b},
$$

and, therefore, its probability density is $\lambda \mathrm{e}^{-\lambda b}$ on $(0, \infty)$. Similarly for $a_{\omega}$ one gets:

$$
\mathbb{P}\left(a_{\omega}<a\right):=\mathbb{P}\{(a, 0) \text { contains no impurities }\}=\mathrm{e}^{-\lambda|a|}=\mathrm{e}^{\lambda a},
$$

and thus its density is $\lambda \mathrm{e}^{\lambda a}$ on $(-\infty, 0)$. Using these distributions in (5.7) we obtain:

$$
\begin{aligned}
\lim _{l \rightarrow \infty} a_{l}(n)= & \mathrm{e}^{n \beta \mu_{\infty}} \lambda^{2} \int_{-\infty}^{0} \mathrm{~d} a \mathrm{e}^{\lambda a} \int_{0}^{\infty} \mathrm{d} b \mathrm{e}^{-\lambda b} \int_{\mathbb{R}} \mathrm{d} x \frac{\mathrm{e}^{-\|x\|^{2}(1 / 2 n \beta+1 / 2 t)}}{\left(4 \pi^{2} \operatorname{tn} \beta\right)^{d / 2}} \\
& \times \int_{\Omega_{(0, x)}^{n \beta}} w^{n \beta}(\mathrm{d} \xi) \chi_{(a, b)}(\xi) \\
= & \mathrm{e}^{n \beta \mu_{\infty}} \lambda^{2} \int_{-\infty}^{0} \mathrm{~d} a \mathrm{e}^{\lambda a} \int_{0}^{\infty} \mathrm{d} b \mathrm{e}^{-\lambda b} \int_{\mathbb{R}} \mathrm{d} x \frac{\mathrm{e}^{-\|x\|^{2}(1 / 2 n \beta+1 / 2 t)}}{\left(4 \pi^{2} t n \beta\right)^{d / 2}} \\
& \times \int_{\Omega_{(0, x)}^{n \beta}} w^{n \beta}(\mathrm{d} \xi) \mathbf{1}\left(\sup _{s}(\xi(s)) \leqslant b\right) \mathbf{1}(\inf (\xi(s)) \geqslant a) \\
= & \mathrm{e}^{n \beta \mu_{\infty} \lambda^{2}} \int_{\mathbb{R}} \mathrm{d} x \frac{\mathrm{e}^{-\|x\|^{2}(1 / 2 n \beta+1 / 2 t)}}{\left(4 \pi^{2} t n \beta\right)^{d / 2}} \\
& \times \int_{\Omega_{(0, x)}^{n \beta}} w^{n \beta}(\mathrm{d} \xi) \int_{-\infty}^{\inf (\xi(s))} \mathrm{d} a \mathrm{e}^{\lambda a} \int_{\sup _{s}(\xi(s))}^{\infty} \mathrm{d} b \mathrm{e}^{-\lambda b},
\end{aligned}
$$

and the Theorem 5.2 follows by explicit computation of the last two integrals.

Proof of Theorem 5.1: Having proved Theorem 5.2, it is now straightforward to derive the corresponding result for the Luttinger-Sy model. Note that the line of reasoning remains unchanged, since only the uniform convergence was used. With these observations, the proof of Theorem 5.1 follows in the same way as for Theorem 4.1 . 
We have proved, in Theorem 5.1, that the Luttinger-Sy model exhibits g-BEC in the kinetic energy states. But, in this particular case, we can go further and determine the particular type of g-BEC in the kinetic energy states. Recall that the g-BEC in the random eigenstates is only in the ground state, that is, of the type I, see (5.5) and [6] for a comprehensive review. Here we shall show that the g-BEC in the kinetic-energy eigenstates is in fact of the type III, namely:

Theorem 5.3 In the Luttinger-Sy model none of the kinetic-energy eigenstates is macroscopically occupied:

$$
\lim _{l \rightarrow \infty} \frac{1}{l}\left\langle N_{l}\left(\psi_{k}\right)\right\rangle_{l}=0 \quad \text { for all } \quad k \in \Lambda_{l}^{*},
$$

even though for $\bar{\rho}>\rho_{c}$ there is a generalized $B E C$.

To prove this theorem we shall exploit the finite-volume localization properties of the random eigenfunctions $\phi_{i}^{\omega, l}$ of the Hamiltonian $h_{l, \mathrm{D}}^{\omega}$. Since the impurities split up the box $\Lambda_{l}$ into a finite number $M^{l}(\omega)$ of sub-intervals $\left\{I_{j}^{\omega}\right\}_{j=1}^{M^{l}(\omega)}$, by virtue of the corresponding orthogonal decomposition of $h_{l, \mathrm{D}}^{\omega}$, cf (5.4), the normalized random eigenfunctions $\phi_{s}^{\omega, l}$ are in fact sine-waves with supports in each of these sub-intervals and thus satisfy:

$$
\left|\phi_{s}^{\omega, l}(x)\right|<\sqrt{\frac{2}{L_{j_{s}}^{\omega}}} \mathbf{1}_{I_{j_{s}}^{\omega}}(x), \quad 1 \leqslant j_{s} \leqslant M^{l}(\omega) .
$$

We require an estimate of the size $L_{j}^{\omega}$ of these random sub-intervals, which we obtain in the following lemma.

Lemma 5.1 Let $\lambda>0$ be a mean concentration of the point Poisson impurities on $\mathbb{R}$. Then eigenfunctions $\phi_{j}^{\omega}$ are localized in sub-intervals of logarithmic size, in the sense that for any $\kappa>4$, one has a. s. the estimate:

$$
\limsup _{l \rightarrow \infty} \frac{\max _{1 \leqslant j \leqslant M^{l}(\omega)} L_{j}^{\omega}}{\ln l} \leqslant \frac{\kappa}{\lambda}
$$

Proof: Define the set

$$
S_{l}:=\left\{\omega: \max _{1 \leqslant j \leqslant M^{l}(\omega)} L_{j}^{\omega}>\frac{\kappa}{\lambda} \ln l\right\} .
$$

Let $n:=[2 \lambda l /(\kappa \ln l)]_{+}$, and define a new box:

$$
\widetilde{\Lambda}_{l}:=\left[-\frac{n}{2}\left(\frac{\kappa}{2 \lambda} \ln l\right), \frac{n}{2}\left(\frac{\kappa}{2 \lambda} \ln l\right)\right] \supset \Lambda_{l} .
$$

Split this bigger box into $n$ identical disjoint intervals $\left\{I_{m}^{l}\right\}_{m=1}^{n}$ of size $\kappa(2 \lambda)^{-1} \ln l$. If $\omega \in S_{l}$, then there exists at least one empty interval $I_{m}^{l}$ (interval without any impurities), and therefore the set

$$
S_{l} \subset \bigcup_{1 \leqslant m \leqslant n}\left\{\omega: I_{m}^{l} \text { is empty }\right\} .
$$

By the Poisson distribution (5.1), the probability for the interval $I_{m}^{l}$ to be empty depends only on its size, and thus

$$
\mathbb{P}\left(S_{l}\right) \leqslant n \exp \left(-\lambda \frac{\kappa}{2 \lambda} \ln l\right) \leqslant\left[\frac{2 \lambda l}{\kappa \ln l}\right]_{+} l^{-\kappa / 2} .
$$

Since we choose $\kappa>4$, it follows that

$$
\sum_{l \geqslant 1} \mathbb{P}\left(S_{l}\right)<\infty
$$


Therefore, by the Borel-Cantelli lemma, there exists a subset $\widetilde{\Omega} \subset \Omega$ of full measure, $\mathbb{P}(\widetilde{\Omega})=1$, such that for each $\omega \in \widetilde{\Omega}$ one can find $l_{0}(\omega)<\infty$ with

$$
\mathbb{P}\left\{\omega: \max _{1 \leqslant j \leqslant M^{l}(\omega)} L_{j}^{\omega} \leqslant \frac{\kappa}{\lambda} \ln l\right\}=1
$$

for all $l \geqslant l_{0}(\omega)$.

Now we can prove the main statement of this section.

Proof of Theorem 5.3: The atom of the measure $\tilde{m}$ has already been established in Theorem 5.1. Concerning the macroscopic occupation of a single state, we have

$$
\begin{aligned}
\frac{1}{l}\left\langle N_{l}\left(\psi_{k}\right)\right\rangle_{l} & =\frac{1}{l} \sum_{i}\left|\left(\phi_{i}^{\omega, l}, \psi_{k}\right)\right|^{2}\left\langle N_{l}\left(\phi_{i}^{\omega, l}\right)\right\rangle_{l} \\
& =\frac{1}{l} \sum_{i}\left\langle N_{l}\left(\phi_{i}^{\omega, l}\right)\right\rangle_{l}\left|\int_{\Lambda_{l}} \mathrm{~d} x \bar{\psi}_{k}(x) \phi_{i}^{\omega, l}(x)\right|^{2} \\
& \leqslant \frac{1}{l} \sum_{i}\left\langle N_{l}\left(\phi_{i}^{\omega, l}\right)\right\rangle_{l} \frac{1}{l}\left(\int_{\Lambda_{l}} \mathrm{~d} x\left|\phi_{i}^{\omega, l}(x)\right|\right)^{2},
\end{aligned}
$$

where in the last step we have used the bound $\left|\psi_{k}\right| \leqslant 1 / \sqrt{l}$. Therefore, by (5.8) and Lemma 5.1, we obtain a. s. the following estimate:

$$
\frac{1}{l}\left\langle N_{l}\left(\psi_{k}\right)\right\rangle_{l} \leqslant \frac{1}{l} \sum_{i}\left\langle N_{l}\left(\phi_{i}^{\omega, l}\right)\right\rangle_{l} \frac{1}{l} \frac{\kappa}{\lambda} \ln l
$$

which is valid for large enough $l$ and for any $\kappa>4$. The theorem then follows by taking the thermodynamic limit.

\section{Acknowledgements}

This paper is an extended version of the talk given by one of the authors (V.A.Z.) at the Conference on Statistical Physics: Modern Trends and Applications (June 23-25, 2009, Lviv, Ukraine) dedicated to the 100th anniversary of N.N. Bogoliubov. We would like to express our thanks to organizers for invitation to give this talk and for their hospitality. 


\title{
References
}

1. Kac M., Luttinger J.M., J. Math. Phys., 1973, 14, 1626-1628.

2. Kac M., Luttinger J.M., J. Math. Phys., 1974, 15, 183-186.

3. Luttinger J.M., Sy H.K., Phys. Rev. A, 1973, 7, 712-720.

4. Lenoble O., Pastur L.A., Zagrebnov V.A., Comptes-rendus de l'Académie des Sciences (Paris), Physique, 2004, 5, 129-142.

5. Zagrebnov V.A., J. Phys. Stud., 2007, 11, 108-121.

6. Lenoble O., Zagrebnov V.A., Markov Processes and related fields, 2007, 13, 441-468.

7. Zagrebnov V.A., Bru J.-B., Phys. Rep., 2001, 350, 291-434.

8. Huang K., Men H.F., Phys. Rev. Lett., 1992, 69, 644-647.

9. Kobayashi M., Tsubota M., Phys. Rev., 2002, 66, 174516.

10. Kirsch W., Martinelli F., Commun. Math. Phys., 1983, 89, 27-40.

11. Simon B., J. Stat. Phys., 1985, 38, 65-76.

12. Pastur L.A., Figotin A. Spectra of Random and Almost-Periodic Operators. Springer-Verlag, Berlin, 1992.

13. van den Berg M., Lewis J.T., Pulé J.V., Helv. Phys. Acta, 1986, 59, 1271-1288.

14. Pulé J.V., Zagrebnov V.A., J. Math. Phys., 2004, 45, 3565-3583.

15. Feller W. An Introduction to Probability Theory and its Applications, Volume II. John Wiley and Sons, New York, 1957.

16. Reed M., Simon B. Methods of Mathematical Physics, IV: Analysis of Operators. Academic Press, London, 1978.

17. Feller W. An Introduction to Probability Theory and its Applications, Volume I. John Wiley and Sons, New York, 1957

18. Pulé J.V., J. Math. Phys., 1983, 24, 138-142.

\section{Бозе конденсація у (випадкових) пастках}

\author{
Т. Яек ${ }^{1}$, Й.В. Пулє ${ }^{1}$, В.А. Загребнов ${ }^{2}$ \\ 1 Школа математичних наук, Університетський коледж Дубліна Белфілд, Дублін 4, Ірландія \\ 2 Середземноморський університет Марсель II, Центр теоретичної фізики, 13288 Марсель, Франція
}

Отримано 15 червня 2009 р.

Проведено дослідження ідеального газу Бозе-частинок у випадкових зовнішніх потенціалах (пастках). Показано, що узагальнена конденсація Бозе-Айнштайна у випадкових власних станах частинок спостерігається тоді і лише тоді, коли це саме стосується і власних станів одночастинкової кінетичної енергії, які відповідають узагальненій конденсації вільного газу Бозе. Крім того, доведено, що значення густини конденсату $є$ однаковим в обох випадках. Це твердження є важливим для підтвердження застосовності наближення Боголюбова в теорії невпорядкованих систем бозонів.

Ключові слова: узагальнена конденсація Бозе-Айнштайна, випадкові потенціали, інтегровані густини станів, “хвости” Ліфшиця

PACS: 05.30.Jp, 03.75.Fi, 67.40.-w 
